\title{
A Brief History of National Surveillance Efforts for Periodontal Disease in the United States
}

\author{
Bruce A. Dye ${ }^{* \dagger}$ and Gina Thornton-Evans ${ }^{\dagger}$
}

National efforts directed toward improving our understanding of the epidemiology of periodontal disease began nearly a half century ago following the development of Russell's periodontal index (PI). United States Public Health Service agencies began national surveillance activities for periodontal disease with the first National Health Examination Survey in 1960 to 1962, and this continued periodically through 2004 in the National Health and Nutrition Examination Survey (NHANES). Periodontal disease status was assessed by using the PI in the earlier national health surveys, but beginning in the 1980s, direct measures for clinical attachment loss were made in national health surveys and continued through 2004 in NHANES. This article provides a general history of the development and implementation of national surveillance efforts for periodontal disease from the mid-1950s to 2005. It also provides brief background information on the factors that have influenced these national surveillance efforts. $J$ Periodontol 2007;78:1373-1379.

\section{KEY WORDS}

Epidemiology; NHANES; oral health; periodontal disease; public health dentistry; surveys.

\footnotetext{
* National Center for Health Statistics, Centers for Disease Control and Prevention, Hyattsville, MD

$\dagger$ University of Maryland Dental School, Baltimore, MD.

† Division of Oral Health, Centers for Disease Control and Prevention, Atlanta, GA.
}

1 n 1980, Dunning ${ }^{1}$ wrote, "The epidemiology of periodontal disease is one of the most important challenges before the dental profession at the moment, but work upon it has been retarded by a number of factors which make it a more difficult subject to study than caries."

Epidemiology is the study of the distribution and determinants of health conditions or events in populations and the application of this study to control health problems. ${ }^{2}$ The application of epidemiology in the study of periodontal disease can be traced back to the early work of A.L. Russell ${ }^{3}$ and the development of the periodontal index (PI) in the 1950s. The genesis of the PI was to address measurement issues inherent to the lack of reliable epidemiological data for periodontal disease and to develop methodology that would promote the surveillance of periodontal disease similar to the already widely used DMFT (decayed, missing, and filled teeth) index, which was introduced by United States Public Health Service dentists Klein, Palmer, and Knutson ${ }^{4}$ in 1938 for dental caries surveillance.

In 1950, Chilton ${ }^{5}$ wrote there was "very little information of a statistically reliable nature on the epidemiologic characteristics of periodontal disease," and he articulated a need for improvements in terminology, measurement, and diagnosis as a remedy. During the following year, Marshall-Day ${ }^{6}$ suggested that periodontal disease was a "serious and universal public health problem," although

doi: 10.1902/jop.2007.060210 
there was a lack of epidemiological data to support such a conclusion. Later, a Periodontal Workshop Committee was convened at the University of Michigan and charged with evaluating the prevention of periodontal disease. ${ }^{7}$ The Committee identified two roles for dental public health, one of which was to promote research related to the epidemiology of periodontal disease, including the development of indices for evaluating periodontal status. A statement originating from this workshop, and quoted later by Russell, ${ }^{3}$ proclaimed "the lack of valid indexes for determining the prevalence and epidemiological characteristics of periodontal diseases had hindered seriously the development of more effective preventive and treatment procedures for these diseases." By 1960, the PI became a prominent measurement tool for surveying periodontal disease prevalence.

\section{THE EARLY YEARS: THE PERIODONTAL INDEX}

The PI criteria were rooted in the prevailing understanding of the etiology and progression of periodontal disease for the time. Russell ${ }^{8}$ stated that the criteria were "based upon the signs of periodontitis and the sequence in which they usually appear - inflammation, pocket formation, and loss of function." Although often referred to as a scaled scoring system, the PI actually was a weighted categorical scoring system. A score of "0" was applied if there was "no overt inflammation," a score of " 1 " was given for "mild gingivitis," a score of " 2 " was given for "gingivitis," a score of "6" was given for "gingivitis with pocket formation," and a score of " 8 " was given for "advanced destruction with loss of function." 3 Each tooth was scored; all scores were summed and divided by the total teeth assessed to produce a mean subject PI score.

To ascribe a level of clinical relevance for the PI, Russell ${ }^{3}$ conducted a study to evaluate the relationship between clinical diagnoses and the PI at the National Institutes of Health (NIH), Clinical Center Dental Clinic. He later advocated that a mean score in the range of 0.1 to 1.0 was indicative of "gingivitis," 1.5 to 5.0 was related to "established destructive periodontal disease," and scores ranging from 4.0 to 8.0 were classified as "terminal disease." 9 The first large-scale epidemiological investigation to use the PI was a group of studies comprising nearly 30,000 Americans residing in 14 cities. ${ }^{10}$ This was followed by a series of international nutrition surveys conducted by the Interdepartmental Committee on Nutrition for National Defense (ICNND) between 1958 and 1961. ${ }^{11}$ The ICNND was an activity coordinated within the $\mathrm{NIH}$ with funding administered by the United States Defense Department's Mutual Defense Assistance Program. ${ }^{12}$ The collection of PI information occurred in eight countries, including the United States; however, domestic ICNND data collection was limited to Alaska.
The application of epidemiological methods to study periodontal disease was now on the dental research community's radar. Kreshover and Russell, ${ }^{13}$ who were senior managers at the National Institute of Dental Research, wrote in the Journal of the American Dental Association in 1958 that research in periodontal disease was continuing to expand and that "it was gratifying to note the increased attention that was given to epidemiologic studies." The authors, referring back to some of the 1951 University of Michigan Periodontal Workshop conclusions, reinforced the notion that effective prevention and therapeutic interventions for periodontal disease would only be realized once practical epidemiological methods for the study of periodontal disease could be developed and implemented.

\section{THE EARLY YEARS: NATIONAL HEALTH SURVEYS}

During the 1950s, there was a growing awareness among public health advocates and policymakers of the lack of national health statistics in the United States; this paucity of health data was considered a major impediment to the development of health promotion and disease prevention activities. In 1955, the Department of Health, Education, and Welfare (DHEW) proposed a plan that would authorize the Surgeon General of the Public Health Service to conduct a "continuing survey of illness and disability" in the United States. ${ }^{14}$ Subsequently, Congress passed the National Health Survey Act as Public Law 652, and the bill was signed by the President on July 3, 1956. The Act was actually a program of surveys: a health interview survey, a health examination survey, and a health records survey. Planning for the first national health interview survey immediately followed the President's signing of the bill into law, and data collection began in 1957.

Planning for the first national health examination survey began in 1957, but full-scale data collection was not undertaken until 1961. The National Health Examination Survey (NHES) was implemented in three "cycles," with each phase representing a specific age range for sample participants. For example, the NHES I was conducted on participants aged 18 to 79 years in 1960 to $1962 .{ }^{15}$ The operationalization of the NHES I was groundbreaking in many aspects for public health surveillance, but maybe more so for the surveillance of the public's oral health. Trained United States Public Health Service dental officers administered standardized dental examinations that included assessing periodontal status using the PI method. The information collected in the NHES I was used to produce the first national estimates of periodontal disease prevalence in the United States. 
These first estimates of periodontal disease prevalence in the United States were weighted to reflect a non-institutionalized dentate population of $\sim 90$ million Americans aged 18 to 79 years. ${ }^{16}$ A 1974 report focusing on the relationship between periodontal disease and selected medical findings from the NHES I concluded that a relationship between periodontal disease, as measured by the PI, and adverse cardiovascular conditions, psychological stress, and diabetes was likely to exist. ${ }^{17}$ Moreover, the authors speculated that "despite the lack of knowledge about the pathogenesis of degenerative periodontal disease, it seems not only possible but indeed likely that any ailment or condition that lowers the body's resistance to infection will favor or perhaps even cause the onset and progression of destructive periodontal disease." A third NHES was conducted during 1966 to 1970 targeting youths aged 12 to 17 years. ${ }^{18}$ Estimates of periodontal disease prevalence were weighted to represent nearly 23 million non-institutionalized youths in the United States. ${ }^{19}$

One major disadvantage of the PI that has become evident with time is the reliance on subjective, rather than objective, measurement of the clinical presentation of disease. The PI scored all periodontal pockets the same, and inflammation was graded subjectively with no apparent differentiation between gingivitis and periodontitis. Because assessments were made visually, pocketing often was based on the severity of gingivitis and tooth mobility. To address some of these issues, Dunning and Leach ${ }^{20}$ proposed the gingival-bone (GB) count, and Ramfjord ${ }^{21}$ introduced the periodontal disease index (PDI) in 1959. The GB count resembled the PI numbering system, but a separate score for gingivitis and bone loss was calculated; Ramfjord's PDI became the first index to use a periodontal probe to measure clinical loss of attachment.

Ramfjord $^{22}$ stated numerous epidemiologically based objectives for the use of the PDI. Among these were to describe the distribution and determinants of periodontal disease and to promote the assessment of treatment needs and the evaluation of prevention activities. To facilitate the use of the PDI in epidemiological studies, Ramfjord selected six teeth to represent the full dentition. The PDI scored the gingival status first using a 0 to 3 scale that was based upon Russell's PI and Schour and Massler's ${ }^{23}$ the gingival papilla, the gingival margin, and the gingival attachment (PMA) gingival index; probing depth measures were made in millimeters, with rounding made to the lower whole number. However, these direct measures were converted to a scale $(4,5$, or 6$)$ based upon the depth of the pocket in relationship to the cemento-enamel junction. Although the PDI was never used to produce national estimates of periodontal disease in the United States, Ramfjord's concept of a partial mouth examina- tion and the indirect method of measuring attachment loss would be used in future national oral health surveys.

As cycle III of the NHES was drawing to a close, the DHEW established a National Nutrition Surveillance System (NNSS) in 1969 to follow-up on concerns of continual malnutrition in the United States and to provide for ongoing measurement of nutrition status of Americans over time. The DHEW proposed that the NNSS should be established under the authority of the National Health Survey Act of 1956 and should be a continuing national probability sample survey that, among other goals, would collect information that could permit the study of nutrition with general health. Consequently, the NNHS was incorporated into the planning stages of a cycle IV for the NHES, and the NHES was renamed as the National Health and Nutrition Examination Survey (NHANES), ${ }^{24}$ which would later be known as NHANES I. Unlike the previous NHES cycles, NHANES would be inclusive of all ages. NHANES I was conducted by the National Center for Health Statistics (NCHS) in 1971 to 1974 on a target population aged 1 to 74 years.

For the assessment of periodontal disease in NHANES I, the PI was used again to promote the evaluation of trends in the epidemiology of periodontal disease between 1960 and 1962 and between 1971 and 1974. The PI was administered on all sampled participants aged 6 to 74 years. This was the only time that national efforts at periodontal disease surveillance included persons $<13$ years of age. The first government report $^{25}$ on selected dental findings from NHANES I highlighted mean PI score disparities between white and black Americans stratified by age. The authors also reported on the severity of periodontal disease, by identifying those with one to three pockets and those with four or more pockets, to show that periodontal disease severity increased with age. For example, 39.7\% of Americans aged 65 to 74 years had four or more pockets compared to $10.3 \%$ of those aged 18 to 44 years.

NHANES II was conducted by NCHS from 1976 to 1980 without an oral health examination. During this period, an expert nutrition panel recommended that NHANES conduct a study of health and nutrition status on a subpopulation of the United States following the conclusion of NHANES II using assessments similar to those employed in NHANES I and NHANES II. ${ }^{26}$ To address the panel recommendations and the inability to produce adequate prevalence estimates for Hispanics from prior national health examination surveys, NCHS conducted the Hispanic Health and Nutrition Examination Survey (HHANES) from 1982 to 1984. Three population subgroups of Hispanics were targeted (Mexicans, Cubans, and Puerto Ricans). An oral health examination similar to NHANES I was used on HHANES, with periodontal disease status assessed by the PI. Findings indicated that Puerto Ricans 
had the highest level of periodontal disease among the Hispanic groups. ${ }^{27}$ With the conclusion of the HHANES, an era of national periodontal disease surveillance using the PI ended in the United States following 2 decades of use.

\section{PERIODONTAL ASSESSMENTS IN TRANSITION}

By the late 1970s, choosing an epidemiological method to assess periodontal disease was becoming increasingly problematic, influenced by an emerging paradigm shift in the understanding of the natural history of periodontal disease. Earlier concepts of periodontal disease assumed a natural progression from gingivitis to periodontitis when left untreated; given the widespread prevalence of gingivitis, nearly everyone was considered at risk for periodontal disease. ${ }^{28}$ Using findings based on individual periodontal site measurements of probing depth and attachment loss, one seminal prospective cohort study ${ }^{29,30}$ challenged the prevailing periodontal disease linear progression theory of untreated gingivitis leading to periodontitis with subsequent tooth loss. Moreover, as researchers began to use the periodontal data from the prior NHES and NHANES for analytical epidemiological studies, concerns started to percolate for identifying an improved practice for periodontal disease surveillance in the United States. It was becoming evident that using a nominal scale to produce an overall PI score for the individual, which was used to calculate population means (an epidemiological advantage in the 1960s), was restricting significantly the study of the distribution of severe disease. Consequently, "more current periodontal disease measures" were advocated for future national surveys to permit analyses directed toward the understanding of the epidemiology of periodontal disease. ${ }^{31}$

Identifying the appropriate level of observation and unit of analysis was another developing measurement issue that was affecting periodontal disease epidemiology in the United States. ${ }^{32}$ The PI scoring system was designed to use teeth as the level of observation and the individual as the unit of analysis. In the late 1970s, the Community Periodontal Index of Treatment Needs (CPITN) was developed and shortly thereafter was endorsed by the World Health Organization (WHO) for population-based surveys. ${ }^{33}$ The CPITN, which later was renamed the Community Periodontal Index by WHO, ${ }^{34}$ was based on observing and scoring 10 index teeth (the four second molars were excluded in persons $<20$ years of age) and calculating a composite individual score for analysis. Unlike the PI, using the CPITN required the examiner to make standard probing depth measures using a special periodontal probe while assigning a sextant score. During this period, many periodontal re- searchers were beginning to advocate site-specific measuring and the reporting of clinical attachment loss as the preferred mechanism for periodontal disease surveillance. This advocacy also paralleled the growing interest among clinical periodontists to use attachment loss as the standard to measure past disease as a surrogate for measuring treatment effects.

\section{THE ASCENDANCY OF ATTACHMENT LOSS MEASURES IN SURVEYS}

These issues converged in the mid-1980s in the United States during planning and implementation of the next series of national health examinations that would include surveillance measures for periodontal disease. In 1985 to 1986 , the National Institute of Dental Research, now known as the National Institute of Dental and Craniofacial Research (NIDCR), conducted the National Survey of Oral Health in United States Employed Adults and Seniors. ${ }^{35}$ The periodontal assessment used in this survey represented a balance between the newer understandings of periodontal disease epidemiology, the outmoded or limited usefulness of periodontal indices, and the available resources. Instead of implementing an index-based system, probing depth measures were recorded in millimeters, and loss of attachment was calculated. The presence of calculus and bleeding from probing also were recorded. These practices led to the collection of disaggregate site-specific information that permitted researchers to derive a variety of periodontal disease case definitions from the same data source. However, this resource-intense data collection procedure required the use of a partial-mouth examination to reduce examination time and related costs. Although United States estimates for attachment loss were calculated for the first time, underestimation of disease prevalence became a possibility with the partial-mouth examination procedure. ${ }^{36}$

In 1988, all of the strengths and limitations of the NIDCR method for periodontal assessment were carried over to the next national health examination survey. NHANES III was conducted by NCHS from 1988 to 1994 , and the oral health component of NHANES III was a collaborative effort between NIDCR and NCHS. The study design of NHANES III was unique compared to prior national health examination surveys. The study was designed to have two nationally representative study periods, or phases, of equal sample size and length. Moreover, the prime goals of NHANES III reflected an expansion of intent beyond providing descriptive information to providing information that would contribute to the understanding of disease etiology and natural history. ${ }^{37}$

The descriptive findings from phase 1 (1988 to 1991) and later from the combined phases (1988 to 1994) 
represented the first reporting of probing depth, gingival recession, and attachment loss in the United States. ${ }^{38,39}$ Although there were significant methodological differences used on NHANES III compared to prior national surveys for periodontal disease assessment that resulted in the inability to investigate trends in periodontal status over the past 3 decades, NHANES periodontal data have been used for two important national surveillance and health promotion-related activities: the Surgeon General's Report on Oral Health ${ }^{40}$ and Healthy People 2010. ${ }^{41}$

In 1999, NHANES was changed from a periodic survey to a continuous, annual survey using a nationally representative sample for each year of data collection. The data are released in 2-year periods to protect confidentiality and to increase statistical reliability. The periodontal assessments used on NHANES beginning in 1999 were virtually unchanged from NHANES III and continued through 2004. From 1999 to 2000 , the same two periodontal sites measured on NHANES III were assessed on NHANES 1999 to 2000. However, a third site, the distal-facial, was added in 2001. Additional information describing the NHANES periodontal methods are available elsewhere. ${ }^{42}$ From 1999 to 2004, the NHANES oral health component was a collaborative effort between NIDCR, the Centers for Disease Control and Prevention (CDC)'s Division of Oral Health, and NCHS.

Because the same protocols were used to assess probing depth and calculate loss of attachment in NHANES III and NHANES 1999 to 2004, evaluating changes in the prevalence of periodontal disease in the United States is possible. A recent report defining periodontal disease, using a combination of probing depth and loss of attachment information, suggested that the prevalence of disease decreased between 1988 to 1994 and 1999 to 2000 among the three major race/ethnic groups observed: Mexican Americans, non-Hispanic blacks, and non-Hispanic whites. $^{43}$

A half a century ago, Chilton ${ }^{5}$ commented that "although no definite data can be presented on the extent of the effects of periodontal disease on the health of the public, it has long been recognized that periodontal disease may have an influence on the general health of the individual." Past efforts by United States Public Health Service agencies that culminated in the current examination procedures for periodontal disease surveillance led to the creation of a remarkably rich set of NHANES data files for periodontal disease research. A number of published analytical papers using NHANES III data have improved our understanding of the epidemiology of periodontal disease and the relationship of periodontal status to systemic health. Some of these reports described the attributable risk of smoking to periodontitis, ${ }^{44}$ the relationship between environmental tobacco smoke and periodontal disease, ${ }^{45}$ an association between attachment loss and history of heart attack, ${ }^{46}$ and the relationship between periodontal health status and cardiovascular risk factors, ${ }^{47,48}$ among many others.

\section{FUTURE CHALLENGES TO ONGOING PERIODONTAL ASSESSMENT IN SURVEYS}

Nearly 20 years ago, the Institute of Medicine issued a report on the "Future of Public Health" describing the core functions of public health as assessment, policy development, and assurance. ${ }^{49}$ The routine collection and dissemination of information on oral health status falls squarely in the assessment function. There are two broad limitations of the NHANES periodontal assessment methods. One involves the use of partialmouth assessments to produce precise estimates for the prevalence of periodontal disease. ${ }^{50-52}$ The other relates to the type of resources required to undertake these partial-mouth assessments. Regarding the latter, trained examiners are required, specialized examination equipment is needed, and appropriate safety and infection control practices must be implemented to operationalize the periodontal examination protocols. Moreover, the time to administer the periodontal examination is highly dependent upon participants' dentate status and comfort level with the probing. The combination of these features represents a critical factor in a survey examination environment, such as NHANES, that requires a typical adult to commit to a 3- to 4-hour examination involving multiple physical examinations and the collection of biologic specimens in a mobile examination center. Consequently, the resource-intense NHANES periodontal assessment presents a challenge for assuring the regular collection and dissemination of periodontal disease information in the United States.

Because of the continual high level of effort required to maintain a dentist-based examination in the annual NHANES, a funding reduction for oral health occurred with the 2005 data collection cycle. Although an oral health examination remains in NHANES, there is no periodontal status assessment. The current NHANES oral health procedures are performed by non-dental professionals using a basic screening method designed to identify easily observable conditions that are reported as present or not present. These procedures permit the CDC to maintain regular data collection for the ongoing surveillance of all of the Healthy People 2010 oral health national objectives that are based on clinical measures, except for periodontal disease. This fuels the impetus to research novel approaches for the surveillance of periodontal disease in the United States. Although there have been many achievements at the national level 
for periodontal disease surveillance over the past half century, once again it must be recognized that "the epidemiology of periodontal disease is one of the most important challenges before the dental profession at the moment."

\section{ACKNOWLEDGMENT}

The findings and conclusions in this report are those of the authors and do not necessarily represent the views of the funding agency.

\section{REFERENCES}

1. Dunning JM. Principles of Dental Public Health, 3rd ed. Cambridge, MA: Harvard University Press; 1980:161.

2. Centers for Disease Control and Prevention. National Center for Chronic Disease Prevention and Health Promotion, Excellence in Curriculum Integration Through Teaching Epidemiology (EXCITE). Available at: http:// www.cdc.gov/excite/library/glossary.htm. Accessed May 3, 2006.

3. Russell AL. A system of classification and scoring for prevalence surveys of periodontal disease. $J$ Dent Res 1956;35:350-359.

4. Klein H, Palmer CE, Knutson JW. Studies on dental caries. I. Dental status and dental needs of elementary school children. Public Health Rep 1938;53:751-765.

5. Chilton NW. Some public health aspects of periodontal disease. J Am Dent Assoc 1950;40:28-33.

6. Marshall-Day CD. The epidemiology of periodontal disease. J Periodontol 1951;22:13-22.

7. Report of Evaluating Committee V. Prevention of periodontal disease, University of Michigan, 1951 Periodontal Workshop. J Am Dent Assoc 1952;45:33-38.

8. Russell AL. The periodontal index. J Periodontol 1967; 38(Suppl.):585-591.

9. Russell AL, Ayers P. Periodontal disease and socioeconomic status in Birmingham, Alabama. Am J Public Health 1960;50:206-214.

10. Russell AL. Some epidemiological characteristics of periodontal disease in a series of urban populations. J Periodontol 1957;28:286-293.

11. Russell AL. International Nutrition Surveys: A summary of preliminary dental findings. J Dent Res 1963; 42:233-244.

12. Berry FB, Schaefer AE. Program of the interdepartmental committee on nutrition for national defense. J Am Dent Assoc 1958;166:775-777.

13. Kreshover SJ, Russell AL. Periodontal disease. Review of 1957 dental research. J Am Dent Assoc 1958;56: 625-627.

14. Origin, Program, and Operation of the US National Health Survey. Vital Health Stat 11965;(1):1-41.

15. Birkner R. Plan and Initial Program of the Health Examination Survey. Vital Health Stat 11965 Jul;(125): $1-43$.

16. Periodontal Disease in Adults: United States 19601962. Vital Health Stat 11 1965;(12):1-30.

17. Selected Examination Findings Related to Periodontal Disease Among Adults: United States 1960-1962. Vital Health Stat 11 1974;(33):1-31.

18. Plan and Operation of a Health Examination Survey of US Youths 12-17 Years of Age. Vital Health Stat 1 $1974 ;(9): 1-80$.
19. Periodontal Disease Among Youths $12-17$ Years: United States 1966-1970. Vital Health Stat 11 1974;(141): 1-28.

20. Dunning JM, Leach LB. Gingival-bone count: A method for epidemiological study of periodontal disease. J Dent Res 1960;39:506-513.

21. Ramfjord SP. Indices for prevalence and incidence of periodontal disease. J Periodontol 1959;30:51-59.

22. Ramfjord SP. The Periodontal Disease Index (PDI). $J$ Periodontol 1967;38:602-610.

23. Schour I, Massler M. Survey of gingival disease using the PMA index. J Dent Res 1948;27:733.

24. Plan and Operation of the Health and Nutrition Examination Survey, US 1971-73. Vital Health Stat 1 1973;(10a):1-46.

25. Kelly JE, Harvey CR. Basic Data on Dental Examination Findings of Persons 1-74 Years: United States 1971-1974. Vital Health Stat 111979 May; (214):1-33.

26. Plan and Operation of the Hispanic Health and Nutrition Examination Survey, 1982-84. Vital Health Stat 11985 Sep;(19):1-429.

27. Ismail AI, Szpunar SM. The prevalence of tooth loss, dental caries, and periodontal disease among Mexican Americans, Cuban Americans, and Puerto Ricans: Findings from HHANES 1982-84. Am J Public Health 1990;80:66-70.

28. Burt BA. The role of epidemiology in the study of periodontal diseases. Periodontol 2000 1993;2:26-33.

29. Löe H, Anerud A, Boysen H, Smith M. The natural history of periodontal disease in man. Study design and baseline data. J Periodontal Res 1978;13:550-562.

30. Löe H, Anerud A, Boysen H, Smith M. The natural history of periodontal disease in man. The rate of periodontal destruction before 40 years of age. J Periodontol 1978;49:607-620.

31. Douglass CW, Gillings D, Sollecito W, Gammon M. National trends in the prevalence and severity of the periodontal diseases. J Am Dent Assoc 1983;107: 403-412.

32. Beck JD, Löe H. Epidemiological principles in studying periodontal diseases. Periodontol 2000 1993;2: 34-45.

33. Ainamo J, Barmes D, Beagrie G, Cutress T, Martin J, Sardo-Infirri J. Development of the World Health Organization (WHO) Community Periodontal Index of Treatment Needs (CPITN). Int Dent $J$ 1982;32: 281-291.

34. World Health Organization. Oral Health Surveys, Basic Methods, 4th ed. Geneva, Switzerland: World Health Organization; 1997:22.

35. US Department of Health and Human Services. National Institutes of Health (1987). Oral Health of United States Adults: The National Survey of Oral Health in U.S. Employed Adults and Seniors: 1985-86. NIH publication no. 87-2868.

36. Kingman A, Morrison E, Löe H. Systematic errors in estimating prevalence and severity of periodontal disease. J Periodontol 1988;59:707-713.

37. Plan and Operation of the Third National Health and Nutrition Examination Survey, 1988-94. Vital Health Stat 11994 Jul;(32):1-407.

38. Brown LJ, Brunelle JA, Kingman A. Periodontal status in the United States, 1988-91: Prevalence, extent, and demographic variation. J Dent Res 1996;75:672683. 
39. Albandar JM, Brunelle JA, Kingman A. Destructive periodontal disease in adults 30 years of age and older in the United States, 1988-94. J Periodontol 1999;70: 13-29.

40. US Public Health Service, Office of the Surgeon General. Oral Health in America: A Report of the Surgeon General. Rockville, MD: US Department of Health and Human Services, U.S. Public Health Service; 2000. NIH publication no. 00-4713.

41. U.S. Department of Health and Human Services. Healthy People 2000. National Health Promotion and Disease Prevention Objectives. Washington, DC: U.S. Government Printing Office; 1990.

42. Dye BA, Barker LK, Selwitz RH, et al.Overview and quality assurance for the National Health and Nutrition Examination Survey (NHANES) oral health component, 1999-2002. Community Dent Oral Epidemiol 2007;35:140-151.

43. Borrell LN, Burt BA, Taylor GW. Prevalence and trends in periodontitis in the USA: From NHANES III to NHANES 1999-2000. J Dent Res 2005;84:924-930.

44. Tomar SL, Asma S. Smoking-attributable periodontitis in the United States: Findings from NHANES III. National Health and Nutrition Examination Survey. $J$ Periodontol 2000;71:743-751.

45. Arbes S, Agustsdottir H, Slade G. Environmental tobacco smoke and periodontal disease in the U.S. Am J Public Health 2001;91:253-257.

46. Arbes SJ, Slade GD, Beck JD. Association between extent of periodontal attachment loss and self-reported history of heart attack: An analysis of NHANES III data. J Dent Res 1999;78:1777-1782.
47. Wu T, Trevisan M, Genco RJ, Falkner KL, Dorn JP, Sempos CT. Examination of the relation between periodontal health status and cardiovascular risk factors: serum total and high density lipoprotein cholesterol, C-reactive protein, and plasma fibrinogen. Am J Epidemiol 2000;151:273-282.

48. Dye BA, Choudhary K, Shea S, Papapanou PN. Serum antibodies to periodontal pathogens and markers of systemic inflammation. J Clin Periodontol 2005;32: 1189-1199.

49. Committee for the Study of the Future of Public Health. Institute of Medicine. The Future of Public Health. Washington, DC: National Academies Press; 1988: 7-8.

50. Slade GD, Beck JD. Plausibility of periodontal disease estimates from NHANES III. J Public Health Dent 1999;59:67-72.

51. Winn DM, Johnson CL, Kingman A. Periodontal disease estimates in NHANES III: Clinical measurement and complex sample design issues. J Public Health Dent 1999;59:73-78.

52. Kingman A, Albandar JM. Methodological aspects of epidemiological studies of periodontal diseases. Periodontol 2000 2002;29:11-30.

Correspondence: Dr. Bruce A. Dye, Centers for Disease Control and Prevention, National Center for Health Statistics, RM 4416, 3311 Toledo Rd., Hyattsville, MD 20782. Fax: 301/458-4029; e-mail: bfd1@cdc.gov.

Accepted for publication September 27, 2006. 